\title{
Kinetic modeling of melt transesterification of diphenyl carbonate and bisphenol-A
}

\author{
Jyh-Ping Hsu*, Jinn-Jong Wong \\ Department of Chemical Engineering, National Taiwan University, Taipei 10617, Taiwan, ROC
}

Received 28 May 2003; received in revised form 2 July 2003; accepted 2 July 2003

\begin{abstract}
The catalytic reaction of the melt transesterification of diphenyl carbonate (DPC) and bisphenol-A (BPA) is investigated theoretically. A reaction mechanism based on the nucleophilic substitution on the carbonyl group of reactants is proposed for the description of the kinetic behavior of the reaction under consideration. Previous models are extended so that the kinetic model derived is not only more physically sound, but also involves a less number of adjustable parameters. The applicability of the present model is justified by fitting it to the available experimental data reported in the literature, and its performance is found to be satisfactory for a wide range of operating conditions. We conclude that melt transesterification of DPC and BPA is of reversible nature; the forward reaction is second order in the concentrations of DPC and catalyst, and the reverse reaction is third order in the concentrations of phenol, oligomer, and catalyst.

(C) 2003 Elsevier Ltd. All rights reserved.
\end{abstract}

Keywords: Transesterification; Diphenyl carbonate; Bisphenol-A

\section{Introduction}

Diphenyl carbonate (DPC) and bisphenol A (BPA) are the main raw materials for the synthesis of polycarbonate (PC). In recent years, because the nonphosgene route for DPC synthesis has reached maturity, polycarbonate synthesis through melt transesterification of DPC and BPA becomes important [1]. Despite of its significance in industrial applications, studies on the relevant reactions involved are still very limited. Losev et al. [2] initiated the study on the kinetics of melt transesterification of DPC and BPA with zinc oxide catalyst under a reduced pressure. They concluded that the reaction rate is first order in the concentration of BPA. Turska and Wro'bel [3,4] investigated the reaction kinetics experimentally under conditions similar to that used by Losev et al. and they found that the reaction rate is third order, proportional to the product of the concentrations of DPC, BPA, and catalyst. Due to the inconsistency between the data reported in these studies, and the lack of information about possible reversible reactions, the models proposed are not widely accepted. Hersh et al. [5] investigated the melt transesterification of

\footnotetext{
* Corresponding author. Tel.: + 886-223637448; fax: + 886-223623040.

E-mail address: jphsu@ccms.ntu.edu.tw (J.P. Hsu).
}

DPC and BPA in a batch reactor with $\mathrm{LiOH} \cdot \mathrm{H}_{2} \mathrm{O}$ catalyst. A reversible reaction mechanism was considered, and they arrived at a third-order kinetic model, which is proportional to the product of the concentrations of DPC, BPA, and catalyst. In subsequent studies, Kim et al. [6,7] proposed a molecular species model to describe the melt transesterification of DPC and BPA, and they found that the transesterification of DPC and BPA could take place even in the absence of catalyst. The kinetic model of Kim et al. $[6,7]$ involved eight parameters, two rate constants and two activation energies for noncatalyzed reactions, and another two rate constants and two activation energies for catalyzed reactions. The activation energies estimated, however, were found to violate thermodynamics rule. The above-mentioned studies lead to kinetic models that are either lack of experimental support or self-contradictory. The problem arises mainly from the fact that an appropriate reaction mechanism has not been proposed.

The melt transesterification of DPC and BPA is a reversible reaction of complicated nature. High performance liquid chromatography (HPLC) analysis revealed that compounds of various molecular weights are present in reacting mixture [6,7]. This implies that developing an appropriate reaction mechanism, although necessary for both fundamental study and practical applications, is 


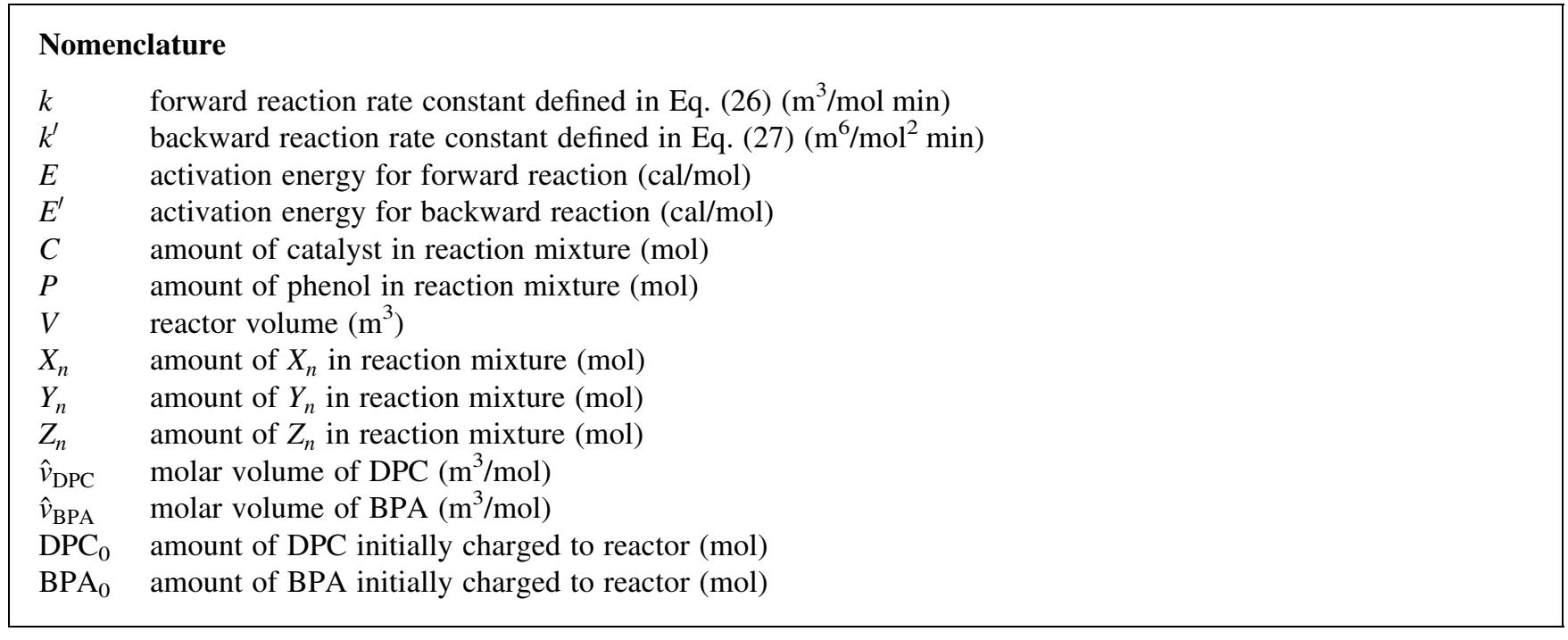

challenging. In this study, the transesterification of DPC and BPA is analyzed theoretically, and an attempt is made to propose a plausible reaction mechanism.

\section{Reaction mechanism}

In the discussion of the synthesis of $\mathrm{PC}$ resin through melt transesterification of DPC and BPA, the synthesis of polyethylene terephthalate (PET) resin through transesterification of dimethyl terephthalate (DMT) and ethylene glycol (EG) is often referred to. The latter uses mainly metallic derivatives as principle catalyst [8-12]; the metal on catalyst surface coordinates to the oxygen atom on the carbonyl group of DMT and activates it for a nucleophilic attack by the oxygen on the hydroxyl group of EG. This leads to a third-order kinetic model in which the reaction rate is proportional to the product of the concentrations of DMT, EG, and catalyst. The mechanism involved in DPCBPA process is different from that in DMT-EG process. They are different, for example, in monomer structure, usage of catalyst, and reaction products. In a DMT-EG process, metallic catalysts such as $\mathrm{Pb}, \mathrm{Zn}$, and $\mathrm{Mn}$ exhibit high activities, but the activities of $\mathrm{Li}, \mathrm{Na}, \mathrm{Ca}$, and $\mathrm{Ba}$ are low. Metallic catalysts can also be used in a DPC-BPA process [13]. The activity of alkali metallic catalyst such as $\mathrm{LiOH}$ and alkali earth metallic catalyst such as $\mathrm{Ba}(\text { acac })_{2}$ are high. On the other hand, that of $\mathrm{Pb}, \mathrm{Zn}$, and $\mathrm{Mn}$ are low. Some nonmetal organic compounds such as pyridine derivatives, imidazole derivatives, and tertiary amines are also used often as catalyst in a DPC-BPA process. These imply that the catalytic mechanism of DPC-BPA process is different from that of DMT-EG process.

DPC molecule contains a carbonyl center connected with two phenolic groups. It is uneasy to carry out exchange directly on the carbonyl center with the hydroxyl group on
BPA, shown as the reaction

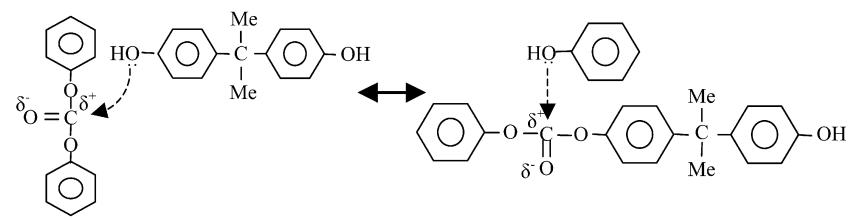

Because transesterification of DPC and BPA hardly takes place in the absence of catalyst [14]; the reaction is very slow even at elevated temperatures, and usage of catalyst is necessary. Since the carbon atom on the carbonyl group brings a small amount of positive charge, alkaline nucleophilic catalyst, which plays the role of nucleophile $(\mathrm{Nu})$, is usually used in carbonyl substitution. Because $\mathrm{Nu}$ is capable of providing electrons (Lewis base) for carbonyl center, it can attack the carbonyl center and form a stable tetrahedral intermediate as shown below:

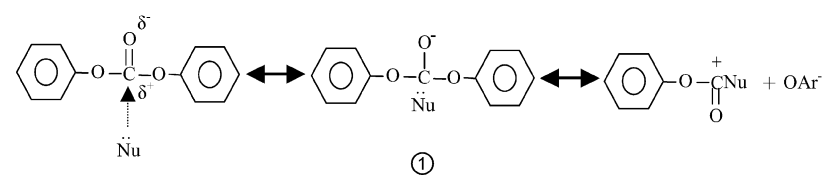

The intermediate may either back to the original reactant or form the product by dropping arlyloxylic group $\left(\mathrm{OAr}^{-}\right)$. For the latter, the follow-up reactions may proceed. The nucleophilic carbonyl substitution expressed in Eq. (2) has a tetrahedral mechanism $[15,16]$, the formation of the tetrahedral intermediate is the rate-determining step. This implies that the reaction is of quadratic nature, and its apparent rate is proportional to the concentration of DPC and $\mathrm{Nu}$. Suppose that the reaction cycle for the catalytic melt transesterification of DPC and BPA can be constructed based on the tetrahedral mechanism expressed in Eq. (2), the basic structure of which is shown in Fig. 1. Under ideal conditions, there are just two end groups in the system, 


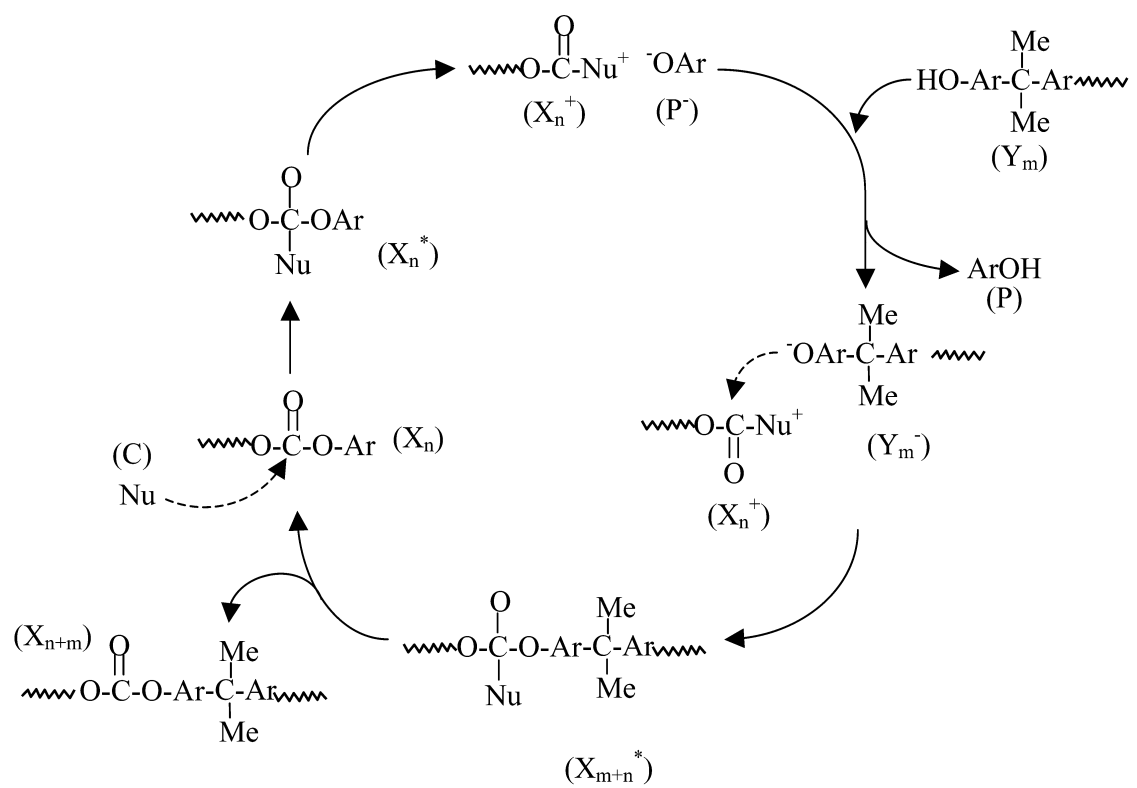

Fig. 1. Proposed reaction mechanism for melt transesterification of diphenyl carbonate and bisphenol-A. $X_{n}$ and $Y_{m}$ represent, respectively, the oligomers with phenyl carbonate groups at both chain terminals and the oligomers with phenyl carbonate group and propane biphenyl group at each chain terminal.

namely, phenyl carbonate group (D group) and 4-hydroxyl2,2-diphenyl propane group (B group). This implies that there are three categories of oligomers: $X_{n}$ with $\mathrm{D}$ groups at both chain terminals, $Y_{n}$ with $\mathrm{D}$ and B groups at each chain terminal, and $Z_{n}$ with $\mathrm{B}$ groups at both chain terminals. When the nucleophilic catalyst $\mathrm{Nu}$ is introduced into the system, it has to attack the carbonyl center of D group to promote the growth of the molecular chain. The attack of $X_{n}$ by $\mathrm{Nu}$ yields intermediate $X_{n}^{*}$. As the arlyloxylic group $\mathrm{P}$ escapes successfully from $X_{n}^{*}$, it will capture proton quickly from its conjugate base $Y_{m}$ (or $Z_{m}$ ), which has B group, followed by the release of phenol. Then, $X_{n}^{+}$and $Y_{m}^{-}$are combined as polymer $X_{n+m}$, which ends the chain growth reaction cycle by releasing $\mathrm{Nu}$. Note that, in addition to the combination of $X_{n}$ and $Y_{m}$, other types of combinations, such as $X_{n}$ and $Z_{m}, Y_{n}$ and $Y_{m}$, and $Y_{n}$ and $Z_{m}$ may also occur simultaneously. As the reaction cycle proceeds, the molecular chain grows, and the molecular weight of the polymer increases until the reaction is complete. The attach of $\mathrm{Nu}$ at a carbonyl center which is not on $\mathrm{D}$ group leads to intermediate $X_{n+m}^{*}$. Because $Y_{m}^{-}$can be regarded as a good leaving group compared to $P^{-}$, its rate of leaving from $X_{n+m}^{*}$ is fast. If phenol molecule exists in the system and the proton is transferred to $Y_{m}^{-}$successfully, then the attack of $\mathrm{Nu}$ promotes the degradation of $X_{n+m}$. On the basis of the mechanism shown in Fig. 1, the following elementary reactions for the combination of $X_{n}$ and $Y_{m}$ can be written:

$X_{n}+\mathrm{Nu} \underset{k_{-1}}{\stackrel{k_{1}}{\rightleftharpoons}} X_{n}^{*}$

$X_{n}^{*} \underset{k_{-2}}{\stackrel{k_{2}}{\rightleftharpoons}} X_{n}^{+}+P^{-}$
$Y_{m}+P^{-} \underset{k_{-3}}{\stackrel{k_{3}}{\rightleftharpoons}} P+Y_{m}^{-}$

$X_{n}^{+}+Y_{m}^{-} \underset{k_{-4}}{\stackrel{k_{4}}{\rightleftharpoons}} X_{n+m}^{*}$

$X_{m+n}^{*} \underset{k_{-5}}{\stackrel{k_{5}}{\rightleftharpoons}} X_{n+m}+\mathrm{Nu}$

The overall reactions are

$X_{n}+Y_{m} \underset{k_{-6}}{\stackrel{k_{6}}{\rightleftharpoons}} X_{n+m}+P$

Similarly, the overall reactions for the combinations of $X_{n}$ and $Z_{m}, Y_{n}$ and $Y_{m}$, and $Y_{n}$ and $Z_{m}$ can be expressed, respectively, as

$X_{n}+Z_{m} \underset{k_{-7}}{\stackrel{k_{7}}{\rightleftharpoons}} Y_{n+m+1}+P$

$Y_{n}+Y_{m} \underset{k_{-8}}{\stackrel{k_{8}}{\rightleftharpoons}} Y_{n+m}+P$

$Y_{n}+Z_{m} \underset{k_{-9}}{\stackrel{k_{9}}{\rightleftharpoons}} Z_{n+m}+P$

It should be pointed out that, although the reactions expressed in Eqs. (8)-(11) were assumed as elementary reactions in the literatures $[6,7]$, they represent only overall stoichiometric relations between reactants and products. Since these equations represent an overall relation between reactants and products, they cannot be used directly to elaborate the reaction mechanisms involved, especially the role of catalyst. The inadequacy of assuming that Eqs. (8)(11) are elementary reactions was justified by that the activation energies estimated based on them violated thermodynamic rule. In subsequent discussions, Eqs. (3)(7) are used to derive our kinetic model. 


\section{Kinetic model}

Let us consider the combination of $X_{n}$ and $Y_{m}$ shown in Fig. 1, where the reactions follow the tetrahedral mechanism. The growth rate of the molecular chain in the reaction cycle is a function of the concentrations of $X_{n}$ and catalyst. The rate-limiting step is the reaction expressed in Eq. (3), the formation of the tetradral intermediate, which is a second-order reaction. The reverse reaction leads to chain degradation. Because it is more difficult for $Y_{m}^{-}$to get proton from phenol than for $P^{-}$to get proton from $Y_{m}$, the reaction expressed in Eq. (5) is the slowest step of the chain degradation. This implies that the rate of the chain degradation is third order, it depends upon the concentrations of $X_{m+n}$, catalyst, and phenol.

For an arbitrary oligomer (or polymer) $X_{n}$, it may be disappeared and born in growth of the molecular chain and in molecular chain degradation. The rate of disappearance of $X_{n}$ in chain growth reaction cycle can be expressed as

$R_{X_{n, a}}=-\frac{2 k}{n+1} \frac{C X_{n}}{V^{2}}, \quad(n \geq 0)$

where $R_{X_{n, a}}$, the rate of disappearance of $X_{n}$ in chain growth reaction, $n$, the chain length, $k$, a proportional constant, which may vary with temperature, $C$, the amount of catalyst in reaction mixture, and $V$, the volume of reactor. For a operation, $V$ can be expressed as

$V=\hat{v}_{\mathrm{DPC}} \mathrm{DPC}_{0}+\hat{v}_{\mathrm{BPA}} \mathrm{BPA}_{0}$

where $\hat{v}_{\mathrm{DPC}}$ and $\hat{v}_{\mathrm{BPA}}$ are, respectively, the molar volume of $\mathrm{DPC}$ and that of $\mathrm{BPA}$, and $\mathrm{DPC}_{0}$ and $\mathrm{BPA}_{0}$ are, respectively, the initial amount of DPC and BPA. If $n=$ 0 , we have $X_{n}=X_{0}=$ DPC. Note that $X_{n}$ molecule has one carbonyl group that can be attacked by catalyst, and $\mathrm{P}^{-}$is capable of leaving the intermediate (1) by two ways, which yields the growth of molecular chain $\mathrm{X}_{1}$. If $n \geqq 1$, there are $(n+1)$ carbonyl groups on $X_{n}$, but the possibility for $\mathrm{P}^{-}$to leave the intermediate remains the same. Therefore, it is reasonable to assume that the reaction rate constant for $R_{X_{n, a}}$ depends upon the length (repeat unit) of the molecular chain. Here, we assume that the rate constant is proportional to $2 /(n+1)$, and is expressed as $2 k /(n+1)$ as that shown on the right-hand side of Eq. (12). Similarly, the rate of production of $X_{n}$ in chain growth reaction cycle of the combination of $X_{n-m}$ and $Y_{m}, R_{X_{n, b}}$, can be expresses as

$R_{X_{n, b}}=\frac{1}{V^{2}} \sum_{m=1}^{n} 2 k C \frac{X_{n-m}}{n-m+1} \frac{Y_{m}}{\sum Y_{i}+2 \sum Z_{i}}$,

$(n \geq 1, m \geq 1)$

The factor $Y_{m} /\left(\sum Y_{i}+2 \sum Z_{i}\right)$ on the right-hand side of this expression can be explained as follows. As indicated in Eqs. (8) and (9), $X_{n-m}$ can either react with $Y_{m}$ to form $X_{n}$, or react with $Z_{m}$ to form $Y_{n+1}$. Suppose that the probabilities of occurrence of these reactions are proportional to the numbers of B-type end groups on the molecules of $Y_{m}$ and $Z_{m}$. Because the total number of B-type end groups is $\left(\sum Y_{i}+2 \sum Z_{i}\right)$, the rate of reaction of $X_{n-m}$ with $Y_{m}$ is proportional to $Y_{m} /\left(\sum Y_{i}+2 \sum Z_{i}\right)$, which is also the fraction of consumed $X_{n-m}$ to form $X_{n}$ through Eq. (8). The coefficient 2 implies that there are two B-type end groups on a $Z_{i}$ molecule. Note that $X_{n}$ may either disappear or form in the molecular chain degradation. The rate of disappearance of $X_{n}$ in chain degradation, $R_{X_{n, c}}$, can be expressed as

$R_{X_{n, c}}=-\frac{(n+1) k^{\prime}}{V^{3}} C X_{n} P, \quad(n \geq 1)$

where $k^{\prime}$, a proportional constant, which may vary with temperature, and $P$, the amount of phenol in reaction mixture. In principle, to know the exact rate expression for the present problem, the rate of degradation of all possible oligomers need to be measured and justified, which is almost impossible at the present stage. In a recent study on the degradation kinetics of poly(bisphenol A carbonate) in benzene, Sivalingam et al. [17,18] assumed a linear dependence of the degradation rate coefficient on the molecular weight of oligomers. A similar approach is adopted here. Because the longer the molecular chain, the faster the degradation, we assume that the rate constant of chain degradation is proportional to chain length, and is equal to $(n+1) k^{\prime}$ as shown on the right-hand side of Eq. (15). The rate of production of $X_{n}$ due to the degradation of $X_{n+m}, R_{X_{n, d}}$, can be expressed as

$R_{X_{n, d}}=\frac{k^{\prime}}{V^{3}} \sum_{l=n+1}^{\infty} \frac{l+1}{l} C X_{l} P, \quad(n \geq 0)$

Because an $X_{\ell}$, molecule has $(\ell+1)$ carbonyl centers, its degradation due to the attack by nucleophilic catalyst may yield $\ell$ possible species, $X_{0}, X_{1}, \ldots, X_{l-1}$. Since $X_{n}$ is one of these species, the rate of production of $X_{n}$ arising from the degradation of $X_{\ell}$ is 1/e the rate of degradation of $X_{\ell}$. Similarly the rate of production of $X_{n}$ arises from the degradation of $Y_{n+m+1}$ in molecular chain degradation, $R_{X_{n, e}}$, can be expressed as

$R_{X_{n, e}}=\frac{k^{\prime}}{V^{3}} \sum_{l=n+1}^{\infty} \frac{l}{2 l-1} C Y_{l} P, \quad(n \geq 0)$

Therefore, the overall reaction rate of $X_{n}$ can be expressed as

$R_{X_{n}}=\frac{1}{V} \frac{\mathrm{d} X_{n}}{\mathrm{~d} t}=R_{X_{n, a}}+R_{X_{n, b}}+R_{X_{n, c}}+R_{X_{n, d}}+R_{X_{n, e}}$

Substituting Eqs. (12) and (14)-(17) into this expression yields

$$
\begin{aligned}
\frac{\mathrm{d} X_{0}}{\mathrm{~d} t}= & -\frac{2 k C X_{0}}{V}+\frac{k^{\prime}}{V^{2}} \\
& \times\left(\sum_{l=1}^{\infty} \frac{(l+1) X_{l}}{l}+\sum_{l=1}^{\infty} \frac{l Y_{l}}{2 l-1}\right) C P
\end{aligned}
$$




$$
\begin{aligned}
\frac{\mathrm{d} X_{n}}{\mathrm{~d} t}= & -\frac{2 k C}{V} \frac{X_{n}}{n+1}+\frac{k C}{V} \\
& \times\left[\sum_{m=0}^{n} \frac{2 X_{n-m}}{n-m+1} \frac{Y_{m}}{\sum Y_{i}+2 \sum Z_{i}}\right] \\
& -\frac{(n+1) k^{\prime}}{V^{2}} C X_{n} P+\frac{k^{\prime}}{V^{2}} \\
& \times\left(\sum_{l=n+1}^{\infty} \frac{(l+1) X_{l}}{l}+\sum_{l=n+1}^{\infty} \frac{l Y_{l}}{2 l-1}\right) C P,
\end{aligned}
$$

$(n \geq 1)$

Similarly, for other species, we have

$$
\begin{aligned}
& \frac{\mathrm{d} Z_{0}}{\mathrm{~d} t}=-\frac{k C}{V}\left[\sum_{m=0}^{\infty} \frac{2 X_{m}}{m+1}+\sum_{m=1}^{\infty} \frac{Y_{m}}{m}\right]\left(\frac{2 Z_{0}}{\sum Y_{i}+2 \sum Z_{i}}\right) \\
& +\frac{k^{\prime}}{V^{2}}\left[\sum_{m=1}^{\infty} \frac{m Y_{m}}{2 m-1}+\sum_{m=1}^{\infty} Z_{m}\right] C P \\
& \frac{\mathrm{d} Z_{n}}{\mathrm{~d} t}=-\frac{k C}{V}\left[\sum_{m=0}^{\infty} \frac{2 X_{m}}{m+1}+\sum_{m=1}^{\infty} \frac{Y_{m}}{m}\right]\left(\frac{2 Z_{n}}{\sum Y_{i}+2 \sum Z_{i}}\right) \\
& +\frac{k C}{V}\left(\sum_{m=0}^{n-1} \frac{Y_{n-m}}{n-m}\right)\left(\frac{2 Z_{m}}{\sum Y_{i}+2 \sum Z_{i}}\right)-\frac{n k^{\prime}}{V^{2}} C Z_{n} P \\
& +\frac{k^{\prime}}{V^{2}}\left(\sum_{m=n+1}^{\infty} \frac{m Y_{m}}{2 m-1}+\sum_{m=n+1}^{\infty} Z_{m}\right) C P \\
& \frac{\mathrm{d} Y_{1}}{\mathrm{~d} t}=-\frac{k C}{V}\left[Y_{1}+\left(\sum_{m=0}^{\infty} \frac{2 X_{m}}{m+1}+\sum_{m=1}^{\infty} \frac{Y_{m}}{m}\right)\right. \\
& \left.\times\left(\frac{Y_{1}}{\sum Y_{i}+2 \sum Z_{i}}\right)-2 X_{0} \frac{2 Z_{0}}{\sum Y_{i}+2 \sum Z_{i}}\right]-\frac{k^{\prime}}{V^{2}} Y_{1} C P \\
& +\frac{k^{\prime}}{V^{2}}\left(\sum_{l=1}^{\infty} \frac{(l+1) X_{l}}{l}+\sum_{l=1}^{\infty} Z_{l}+\sum_{l=2}^{\infty} \frac{2 l Y_{l}}{2 l-1}\right) C P \\
& \frac{\mathrm{d} Y_{n}}{\mathrm{~d} t}=-\frac{k C}{V} \frac{Y_{n}}{n}-\frac{k C}{V}\left(\sum_{m=0}^{\infty} \frac{2 X_{m}}{m+1}+\sum_{m=1}^{\infty} \frac{Y_{m}}{m}\right) \\
& \times\left(\frac{Y_{n}}{\sum Y_{i}+2 \sum Z_{i}}\right)+\frac{2 k C}{V} \sum_{m=0}^{n-1} \frac{X_{n-m-1}}{n-m} \\
& \times \frac{2 Z_{m}}{\sum Y_{i}+2 \sum Z_{i}}+\frac{k C}{V} \sum_{m=1}^{n-1} \frac{Y_{n-m}}{n-m} \\
& \times \frac{Y_{m}}{\sum Y_{i}+2 \sum Z_{i}}-\frac{n k^{\prime}}{V^{2}} Y_{n} C P+\frac{k^{\prime}}{V^{2}} \\
& \times\left(\sum_{l=n}^{\infty} \frac{(l+1) X_{l}}{l}+\sum_{l=n}^{\infty} Z_{l}+\sum_{l=n+1}^{\infty} \frac{2 l Y_{l}}{2 l-1}\right) C P,
\end{aligned}
$$

$(n \geq 2)$

$$
\begin{aligned}
\frac{\mathrm{d} P}{\mathrm{~d} t}= & \frac{k C}{V}\left(\sum_{m=0}^{\infty} \frac{2 X_{m}}{m+1}+\sum_{m=1}^{\infty} \frac{Y_{m}}{m}\right)-\frac{k^{\prime}}{V^{2}} \\
& \times\left(\sum_{m=1}^{\infty}(m+1) X_{m}+\sum_{m=1}^{\infty} m Y_{m}+\sum_{m=1}^{\infty} m Z_{m}\right) C P
\end{aligned}
$$

The set of simultaneously differential equations, Eqs. (12)(25), summarizes the present molecular species model for the catalytic melt transesterification of DPC and BPA. The temperature dependence of the proportional constants $k$ and $k^{\prime}$ is assumed to follow Arrhenius law as

$k=k_{0} \exp (-E / R T)$

$k^{\prime}=k_{0}^{\prime} \exp \left(-E^{\prime} / R T\right)$

where $k_{0}$ and $k_{0}^{\prime}$ are proportional constants, $E$ and $E^{\prime}$ are the activation energies, $R$, gas constant, and $T$, the absolute temperature.

\section{Justification of kinetic model and discussion}

The applicability of the kinetic model derived is justified by fitting it to the available experimental data in the literature [7-9]. The governing equations expressed in Eqs. (12)-(25) are solved numerically by Visual Fortran V5. The experimental conditions and the estimated values of the adjustable parameters are summarized in Table 1. The present kinetic model contains four adjustable parameters, namely, $k_{0}, k_{0}^{\prime}, E$, and $E^{\prime}$; considering the complicated nature of the transesterification reaction, it is parsimonious. It should be pointed out, however, that the actual reaction may involve many other reacting species, and various types of parallel and/or sequential reactions may occur simultaneously in the system. Therefore, the values of the adjustable parameters estimated should be interpreted as mean or apparent values. The values of the adjustable parameters summarized in Table 1 lead to $k=(2.762 \pm$ $0.008) \times 10^{7} \exp (-48,420 \pm 79 / R T) \quad\left(\mathrm{m}^{3} / \mathrm{mol} \mathrm{min}\right)$ and $k^{\prime}=(2.756 \pm 0.007) \times 10^{4} \exp (-24,270 \pm 87 / R T) \quad\left(\mathrm{m}^{6} /\right.$ $\mathrm{mol}^{2} \mathrm{~min}$ ). These values are different appreciably from those reported by Hersh and Choi [5].

Fig. 2 shows the experimental data reported by Hersh and Choi [5] where the temporal variation of phenol concentration at various $\mathrm{LiOH} \cdot \mathrm{H}_{2} \mathrm{O}$ catalyst concentrations were recorded. Both their experimental results and the values based on the present kinetic model are illustrated. Fig. 3 shows another set of experimental data [6,7], where the temporal variation of BPA are recorded at various catalyst concentrations at $210{ }^{\circ} \mathrm{C}$. Figs. 2 and 3 show that the performance of the present model is satisfactory. Note that the experimental conditions considered in these figures cover a wide range of operating temperature and catalyst concentration. In the derivation of the present kinetic model, we assume that the rate equation for the forward reaction of chain growth reaction is second order, which depends on the 
concentrations of DPC and catalyst, and the revere reaction is third order, which depends on the concentrations of phenol, oligomer, and catalyst. Therefore, the role that the main monomer BPA plays is implicit. As can be seen from Fig. 4, the present kinetic model is applicable for a wide range of monomer ratio, temperature, and catalyst concentration.

One of the usages of the molecular species model assumed is to provide information about the variation of each chemical species in the system. This is essential for the prediction of the properties of the final product based on specified operating conditions. Fig. 5, for example, shows the temporal variations in the concentrations of monomers, oligomer and phenol. As can be seen from this figure, the present kinetic model is successful in predicting the general trend of the experimental data. Quantitatively, the predicted rate of consumption of monomers is lower than the corresponding experimental value. This may arise from the fact that the reaction temperature, $230{ }^{\circ} \mathrm{C}$, is much higher than the boiling point of phenol, that is, some of the phenol produced during the reaction is present in the vapor phase. The reaction in the liquid phase is not truly a batch process before the reaction reaches equilibrium state. This leads to a faster rate of reaction.

Fig. 6 shows the temporal variations in the concentrations of oligomer of various molecular weights. Initially, only $X_{0}$ (DPC), $Z_{0}$ (BPA), and catalyst are present in the system. The combination of $X_{0}$ and $Z_{0}$ yields a rapid increase in $Y_{1}$ in the early stage of the reaction. After passing through a maximal value, it decreases to an equilibrium value, and at the same time the concentrations of $X_{1}$ and $Z_{1}$ increase. The qualitative behavior of $X_{1}$ and $Z_{1}$ as time proceeds is similar to that of $Y_{1}$. In general, the performance of the present model is satisfactory.

As mentioned previously, transesterification of DPC and BPA is of complicated nature; various types of serial/parallel reactions may occur, and intermediates, which arise from these reactions, may be present. In practice, it is almost impossible to identify the reactions that actually occur and to estimate the associate rate constants. Here, we assume that the rate constant correlates to the chain length of reaction mixture based on the idea of collision theory. Needless to say, the performance of the kinetic model derived can be improved if a more precise rate expression is available.

\section{Conclusions}

In summary, a reaction mechanism for the melt transesterification of DPC and BPA is proposed. On the basis of a tetrahedral mechanism, the reaction scheme can be constructed, the elementary reactions be written, and a novel molecular species model be deduced. Although the reaction under consideration is of complicated nature, the kinetic model derived contains only four adjustable parameters. By fitting it to the available experimental data reported in the literature, we show that the performance of our model is satisfactory for a wide range of operating conditions. According to the model, melt transesterification of DPC and BPA is of reversible nature; the forward reaction is second order, the rate of which is proportional to the concentrations of DPC and catalyst, and the reverse reaction is third order, the rate of which is proportional to the concentrations of phenol, oligomer, and catalyst.

\section{Acknowledgements}

This work is supported by the National Science Council of the Republic of China.

\section{Appendix A}

\section{Abbreviations for molecular formulas}

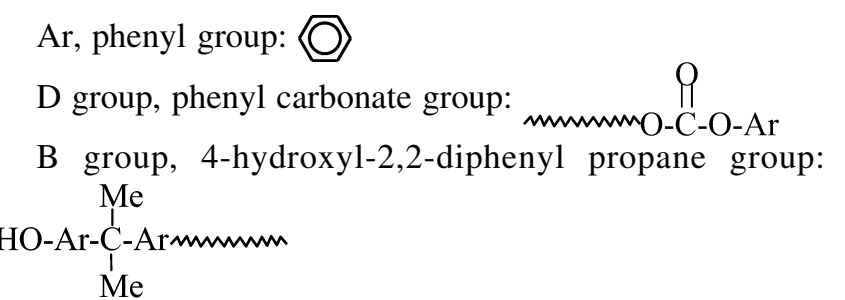

DPC, diphenyl carbonate: $\prod_{\text {Ar-O-C-O-Ar }}$

BPA, bisphenol A, 4,4-dihydroxy-diphenyl-2,2-propane:<smiles>[Y4]c1ccc(Oc2ccc(C(C)(C)c3ccc(OC(C)(C)C(=O)Oc4ccc(C5(C(=O)O)CCC5)cc4)cc3)cc2)cc1</smiles><smiles>[Y17][C@H](C)Oc1ccc(C(C)(C)c2ccc(Oc3ccccc3)cc2)cc1</smiles>

$Z_{n}$<smiles>CC(C)Oc1ccc(C(C)(C)c2ccc(OC3(C(=O)Oc4ccc(C(C)(C)c5ccc(O)cc5)cc4)COC3)cc2)cc1</smiles>

\section{References}

[1] Komiya K, Fukuoka S, Aminaka M, Hasegawa K, Hachiya H. Okamato H, Watanabe T, Yoneda H, Fukawa I, Dozono T, Jn: Anastas PT, Williamson TC editors. ACS Symposium Series: 626, Washington, DC: ACS; 1996. p. 20-32. 
[2] Losev P, Smirnova OV, Smurova YV. Polym Sci USSR 1963;5: 662-70.

[3] Turska E, Wro'bel AM. Polymer 1970;11:408-14.

[4] Turska E, Wro'bel AM. Polymer 1970;11:415-20.

[5] Hersh SN, Choi KY. J Appl Polym Sci 1990;41:1033-46.

[6] Kim Y, Choi KY, Chamberlin TA. Ind Engng Chem Res 1992;31: 2118-27.

[7] Kim Y, Choi KY. J Appl Polym Sci 1993;49:747-64.

[8] Fontana CM. J Appl Polym Sci, A1 1968;6:2343-58.

[9] Wolf KH, Küster B, Herlinger H, Tschang CJ. Die Angew Makromol Chemie 1978;68:23-37.

[10] Otton J, Ratton S. J Appl Polym Sci, Polym Chem Ed 1988;26: $2199-224$.

[11] Santacesaria E, Trulli F, Minervini L, Di Serio M, Tesser R, Contessa S. J Appl Polym Sci 1994;54:1371-84.
[12] Tomita K, Ida H. Polymer 1975;16:185-90.

[13] Ignatov VN, Tartari V, Carraro C, Pippa R, Nadali G, Berti C, Fiorini M. Macromol Chem Phys 2001;202(9):1941-5.

[14] Schnell H. Chemistry and physics of polycarbonates. Polymer reviews: 9, New York: Interscience; 1964.

[15] March J. Advanced organic chemistry: reaction, mechanism, and structure. New York: Wiley; 1992. p. 330-35.

[16] Moore JW, Pearson RG. Kinetics and mechanism, 3rd ed. New York: Wiley; 1981. p. 347.

[17] Sivalingam G, Madras G. Ind Engng Chem Res 2002;41: $5337-40$.

[18] Sivalingam G, Nagaveni K, Madras G, Hegde MS. Ind Engng Chem Res 2003;42:687-91. 